ISSN 0103-5150

Fisioter. Mov., Curitiba, v. 24, n. 2, p. 347-355, abr./jun. 2011 Licenciado sob uma Licença Creative Commons

\title{
Benefícios e riscos da prática de atividade física recreativa e/ou esportiva por pessoas com epilepsia
}

\author{
Benefits and risks of the practice of leisure and/or sportive physical \\ activity by people with epilepsy
}

\author{
Rodrigo Luiz Vancini ${ }^{[a]}$, Claudio Andre Barbosa de Lira ${ }^{[b]}$, Fúlvio Alexandre Scorza ${ }^{[c]}$, \\ Ricardo Mario Arida ${ }^{[\mathrm{d}]}$ \\ [a] Doutor, Departamento de Fisiologia da Universidade Federal de São Paulo (UNIFESP), São Paulo, SP - Brasil, e-mail: \\ rodrigoluizvancini@gmail.com \\ [b] Doutor, Departamento de Fisiologia da Universidade Federal de São Paulo (UNIFESP), campus de Jataí da Universidade \\ Federal de Goiás (UFG), Jataí, GO - Brasil, e-mail: andre.claudio@gmail.com \\ [c] Doutor, Departamento de Neurologia/Neurocirurgia da UNIFESP, São Paulo, SP - Brasil, e-mail: scorza.nexp@epm.br \\ [d] Doutor, Departamento de Fisiologia da UNIFESP, São Paulo, SP - Brasil, e-mail: arida.nexp@epm.br
}

\section{Resumo}

A epilepsia é o distúrbio neurológico crônico mais comum no mundo. 0 tratamento farmacológico é essencial na maioria dos casos. Entretanto, terapias não farmacológicas, como a prática de atividade física regular, vêm sendo estudadas para o tratamento complementar desse distúrbio. Já está bem estabelecido que programas de atividade física promovem benefícios sobre a aptidão física e a saúde. Contudo, pessoas com epilepsia frequentemente são desencorajadas a participar desses programas. Essa relutância origina-se da proteção excessiva dos profissionais da saúde e familiares, pois existe o receio que a prática de atividade física possa predispor os indivíduos a lesões traumáticas ou que a fadiga resultante do esforço físico possa precipitar uma nova crise epiléptica. Evidências crescentes mostram que a prática de atividade física é benéfica para pessoas com epilepsia, havendo poucos achados mostrando o aumento da frequência de crises ou do risco de lesão quando a doença está farmacologicamente controlada. Portanto, o objetivo da presente revisão é apresentar os possíveis riscos e benefícios da prática de atividade física por pessoas com epilepsia.

Palavras-chave: Epilepsia. Crise epiléptica. Exercício. Esporte. 
Abstract

Epilepsy is the most common chronic neurological disorder in the world. Pharmacological treatment is essential in the majority of cases. However, nonpharmacologic therapies such as regular physical activity have been studied in the treatment of epilepsy. It was well established that the regular physical activity provides benefits on physical fitness and health. However, people with epilepsy are often discouraged from participating in physical activity programs. This reluctance derived from overprotection of health's professionals and relatives, because there are fears that the practice of physical activity may predispose individuals to traumatic injury or that fatigue resulting from physical effort can precipitate a seizure. Growing evidence shows that regular physical activity is beneficial for individuals with epilepsy, with few findings showing an increase in seizure frequency or risk of injury when the disease is pharmacologically controlled. Therefore, the purpose of this review is to present the possible risks and benefits of regular physical activity for people with epilepsy.

Keywords: Epilepsy. Epileptic seizure. Exercise. Sport.

\section{Introdução}

A epilepsia é o distúrbio neurológico crônico mais comum no mundo (1-3), sendo definida como a predisposição em gerar crises epilépticas na presença de condições neurobiológicas, psicológicas, cognitivas e sociais específicas. Uma crise epiléptica é a ocorrência transitória de sinais e/ou sintomas em decorrência da atividade neuronal anormal e excessiva (4).

De acordo com a proposição mais atual da International League Against Epilepsy (Ilae), as crises são consideradas condições diagnósticas divididas em três subgrupos: 1) crises isoladas (generalizadas ou focais); 2) crises contínuas, configurando o status epilepticus (condição na qual o cérebro está em estado de crises persistentes) generalizado ou focal; e 3) crises reflexas, em que os fatores precipitantes podem desencadear crises focais ou generalizadas. Resumidamente, as crises generalizadas são aquelas nas quais as descargas epilépticas envolvem simultaneamente os dois hemisférios cerebrais desde o início, enquanto nas crises parciais a atividade epiléptica está limitada a uma área do cérebro. A atividade epiléptica das crises parciais, simples ou complexas pode se difundir tornando-se generalizada e, nesse caso, a crise é denominada secundariamente generalizada $(5,6)$.

Diversos fatores têm sido relacionados como desencadeadores de crises. Dentre esses fatores podemos citar: os estados febris, as mudanças hormonais advindas do ciclo menstrual, o consumo excessivo de cafeína, a privação de sono (7), a abstinência de álcool ou de outras drogas sedativas (8), a exposição à luz estroboscópica (9), o estresse emocional (10), a fadiga (11), a hipóxia (12), a hiper-hidratação (13, 14), a hiponatremia $(15,16)$, a hipertermia (17), a hipoglicemia (18), a hiperventilação voluntária (19) e a manobra de Valsalva (9).

A incidência da epilepsia varia em função da idade, com as maiores taxas ocorrendo na infância, caindo para níveis mais baixos na idade adulta e aumentando novamente ao redor dos 65 anos (3). A epilepsia provoca repercussões socioeconômicas importantes $(20,21)$ na medida em que aumenta os custos econômicos diretos, provenientes dos gastos médicos com drogas e hospitalizações, e indiretos, pela perda de capacidade produtiva, desemprego, licença médica ou morte prematura $(22,23)$. Na América do Norte, por exemplo, os gastos diretos e indiretos com a epilepsia somam 12,5 bilhões de dólares americanos por ano (24).

Para o diagnóstico da epilepsia, a pessoa deve ser avaliada por um médico neurologista que será responsável em determinar a causa principal da doença, assim como a probabilidade da ocorrência de futuras crises. 0 diagnóstico inclui um cuidadoso histórico clínico, exame físico com avaliação neurológica detalhada, testes sanguíneos para determinação de uma possível causa metabólica, eletroencefalograma (EEG) e, frequentemente, exames de imagem cerebral $(9,25)$.

0 tratamento farmacológico, com as drogas antiepilépticas (DAEs), é essencial na maior parte dos casos $(2,26)$. Cerca de metade das pessoas que fazem o uso correto e regular das DAEs permanecem 
livres de crises, sendo o tratamento cirúrgico raramente necessário $(9,25,26)$. Adicionalmente, a boa nutrição associada com o sono e repouso adequado auxiliam no tratamento. Nesse contexto, a utilização de terapias complementares para o tratamento da epilepsia, como a prática de exercício físico, tem sido utilizada (27-29) e pesquisada tanto em modelos experimentais quanto em humanos (27-35). Portanto, o objetivo da presente revisão é apontar os possíveis riscos e benefícios da prática de atividade física regular, recreativa ou desportiva por pessoas com epilepsia.

\section{Epilepsia e atividade física}

Apesar de a prática de atividade física ser enfatizada na sociedade atual pelos benefícios que proporciona sobre a aptidão física e saúde dos indivíduos saudáveis e naqueles com diferentes tipos de doenças, indivíduos com epilepsia são frequentemente desencorajados e muitas vezes excluídos da participação em programas de atividade física (14, 28, 36, 37). Essa relutância origina-se da proteção excessiva dos médicos e familiares, pelo medo que a prática de atividade física possa piorar seu estado, predispor os indivíduos a lesões traumáticas ou que a fadiga resultante do esforço físico possa precipitar uma nova crise $(36,38,39)$.

Além do mais, a principal preocupação das pessoas com epilepsia com relação à prática de atividade física tem sido a possibilidade de essa atividade atuar como um fator indutor ou aumentar a frequência de crises (7). De fato, vários estudos investigaram os hábitos de atividade física e social de pessoas com epilepsia por meio de questionários e/ou estudos clínicos (21, 40-49). A conclusão principal de alguns desses estudos é que a ausência da prática de atividade física por essas pessoas tem relação com as condições de facilidade e acesso ao treinamento físico, os problemas de transporte e deslocamento, a redução da energia pelos efeitos colaterais das DAEs, o medo das crises e de seus possíveis danos e a ausência de profissionais qualificados (41, $43,45,47,50$ ).

Essa ausência da prática de atividade física entre as pessoas com epilepsia conduz a uma baixa aptidão física (41), fazendo com que essas pessoas entrem em um ciclo vicioso: hipoatividade - destreinamento - deterioração funcional - hipoatividade adicional (51), com consequente prejuízo da qualidade de vida (1). Além do mais, o sedentarismo pode conduzir pessoas com epilepsia a aumentar o risco de desenvolverem hipertensão, diabetes, doenças cardíacas $(52)$ e obesidade $(7,48)$, o que piora ainda mais a qualidade de vida e o estado de saúde dessas pessoas. Entretanto, evidências crescentes sugerem que a prática regular de atividade física é benéfica para pessoas com epilepsia, havendo poucos achados mostrando o aumento da frequência de crises ou do risco de lesões quando a doença está controlada (36).

Por exemplo, Camilo et al. (53) investigaram o efeito do exercício físico incremental até a exaustão, por meio do teste ergométrico em pessoas com epilepsia, e demonstraram que nenhum indivíduo apresentou crises durante o esforço físico ou no período de recuperação após o teste. Além disso, os sujeitos apresentaram respostas fisiológicas normais da frequência cardíaca e pressão arterial durante os diferentes estágios do teste de esforço. A conclusão desse estudo sugere que o esforço físico exaustivo parece não ser um componente indutor de crises epilépticas. Vancini et al. (54) avaliaram 20 pessoas com epilepsia do lobo temporal e demonstraram que, apesar destas apresentarem menor aptidão aeróbia com relação à população geral, não há peculiaridades nas respostas fisiológicas durante o exercício físico incremental até a exaustão que requeiram um diferencial de cuidados na avaliação da aptidão física aeróbia dessas pessoas. Além disso, a prática de uma sessão de exercício físico intenso foi capaz de reduzir as descargas epileptiformes no EEG durante o esforço físico e na recuperação após o esforço quando comparado com a condição de repouso.

As crises epilépticas raramente ocorrem durante a prática de atividade física, estando presentes apenas em casos específicos (55-58). Na maioria dos casos, a prática de atividade física diminui o risco das crises, ajudando a controlá-las (7, 40, 58-60). No estudo de Frucht et al. (61), de 400 pessoas com epilepsia avaliadas, apenas duas tinham crises associadas com a prática de atividade física. Bennett (38) sugere que as crises parciais complexas, com ou sem generalização secundária, seriam o tipo de crise mais suscetível de ativação pelo exercício físico e que o lobo temporal, quando comparado a outras áreas corticais, seria mais sensível às descargas epileptiformes induzidas pelo exercício físico. 
0 autor também faz menção ao fato de que se a primeira crise ocorrer durante o esforço físico, particularmente uma crise parcial simples ou complexa, é possível que haja uma causa sintomática, como tumor cerebral ou má-formação arteriovenosa. Essas observações foram sustentadas por outro estudo, que avaliou três adultos com crises generalizadas ocorrendo depois de corrida de baixa intensidade. Os estudos de neuroimagem revelaram lesões corticais frontais em todos os sujeitos avaliados. Os autores desse estudo recomendaram que todas as pessoas com crises após o esforço físico sejam investigadas quanto à presença de pequenas lesões cerebrais, particularmente no córtex frontal (62).

Vale mencionar que pessoas com epilepsia, como consequência das crises e do uso crônico de DAEs, têm maior predisposição a desenvolverem depressão (63) e a prática de atividade física parece ajudar a minimizar esse quadro (42), pois ela melhora o estado de humor (60) e reduz o estresse e a ansiedade.

Existem evidências que atribuem a redução do estresse e da ansiedade ao metabolismo das monoaminas e/ou à liberação de $\beta$-endorfinas, que sabidamente aumentam no sistema nervoso central durante a prática de atividade física, proporcionando sensação de bem-estar (64) e influindo positivamente na qualidade de vida. Sugere-se que o aumento das $\beta$-endorfinas durante a prática de atividade física possa atuar como um anticonvulsivante endógeno natural, inibindo, dessa forma, a atividade epiléptica $(39,65)$.

A prática regular de atividade física pode ser fundamental, pois pessoas com epilepsia possuem distúrbios psicossociais e uma taxa de suicídio cinco vezes maior que a população geral (66). Restringir uma criança ou um adulto da prática de atividade física e esportiva pode conduzir ao isolamento social e a uma baixa autoestima. 0 risco da prática de atividade física, mesmo na presença de crises recorrentes, deve ser pesado contra o trauma psicológico resultante da sua restrição, na maioria das vezes desnecessária (67).

\section{Efeitos do treinamento físico em pessoas com epilepsia}

A participação de pessoas com epilepsia em programas de treinamento físico para a melhora da aptidão física de forma recreativa e/ou competitiva vem sendo debatida há muito tempo. Foi observado que pessoas com epilepsia estão menos propensas a ter crises quando estão ativamente ocupadas, isto é, durante a atividade mental e física, do que quando estão inativas. Outro fator que reduziria a frequência ou a indução das crises seria o estado de alerta (25) e o limiar de vigilância, presentes durante a realização do exercício físico (68).

Nesse contexto, alguns estudos verificaram os efeitos de um programa de treinamento físico em pessoas com epilepsia. Nakken et al. (69) estudaram 21 pacientes com epilepsia não controlada que participaram de um programa de treinamento físico (45 minutos, três vezes por dia, seis vezes na semana), durante 4 semanas, em uma intensidade de no mínimo $60 \%$ do consumo máximo de oxigênio $\left(\mathrm{VO}_{2} \mathrm{máx}\right)$. Esse programa induziu a um aumento de $19 \%$ no $\mathrm{VO}_{2}$ máx, como também a benefícios psicológicos, demonstrando que indivíduos com epilepsia podem ter os mesmos efeitos positivos decorrentes de um programa de exercício físico que qualquer outra pessoa, como: aumento da capacidade aeróbia, frequência cardíaca reduzida para a mesma carga de trabalho, redução da gordura corporal e aumento da autoestima.

Eriksen et al. (52) realizaram um programa de atividade física (dança, treinamento resistido e alongamento) em 15 mulheres com epilepsia, farmacologicamente refratária, durante 15 semanas, 2 vezes na semana, com 60 minutos de duração por sessão. Uma redução na frequência de crises foi observada durante o período de intervenção. 0 programa de exercício físico também contribuiu para a redução das dores musculares, dos distúrbios do sono, da fadiga, do colesterol plasmático, assim como para um aumento significativo de $8 \%$ do $\mathrm{VO}_{2}$ máx. Esse aumento do $\mathrm{VO}_{2}$ máx poderia diminuir as mudanças metabólicas produzidas por fatores estressores da vida diária, pois o disparo de qualquer célula nervosa é influenciado pelo ambiente bioquímico. Nesse sentido, qualquer alteração nesse ambiente pode influenciar a taxa de disparo de uma célula epileptogênica (70). Pessoas com maior aptidão aeróbia necessitam de menor ativação simpática do que pessoas menos aptas para desempenhar a mesma carga absoluta de trabalho, o que contribuiria na diminuição da frequência de crises (71).

McAuley et al. (72) realizaram um programa de exercício físico supervisionado de 12 semanas, com 
3 sessões semanais, em 28 pessoas com epilepsia. Foram avaliadas as variáveis comportamentais (bem-estar emocional, percepção do estado de saúde, raiva, depressão, vigor e competência para prática esportiva), clínicas (frequência de crises epilépticas) e fisiológicas (força muscular, percentual de gordura corporal e $\mathrm{VO}_{2}$ máx). Observou-se que o programa de exercício físico influenciou positivamente as variáveis comportamentais, não produziu alteração na frequência de crises e promoveu benefícios significativos sobre as variáveis fisiológicas, como aumento de $26 \%$ na força muscular, aumento de $12 \%$ no $\mathrm{VO}_{2}$ máx, aumento de $89 \%$ no tempo de exaustão na esteira e diminuição de $11 \%$ no percentual de gordura corporal.

Heise et al. (63) avaliaram o impacto de 12 semanas de treinamento físico em pessoas com epilepsia. Para isso foram selecionadas aleatoriamente 28 pessoas, sendo divididas em grupo exercício e controle. As pessoas do grupo exercício realizaram um programa de treinamento que consistiu na combinação de exercício aeróbio e de força, três vezes por semana, aproximadamente uma hora por dia. 0 grupo controle foi instruído a manter seu nível habitual de atividade física ao longo de todo o período de estudo. Todos os participantes foram avaliados do ponto de vista fisiológico, comportamental e clínico antes e após as 12 semanas de treinamento físico. 0 grupo exercício apresentou aumento significativo da força muscular, do $\mathrm{VO}_{2}$ pico e do tempo de resistência na esteira durante a realização de um exercício submáximo após as 12 semanas de treinamento físico, assim como diminuição significativa da gordura corporal, do colesterol total, dos níveis de triglicérides e da lipoproteína de baixa densidade. Além disso, o programa de treinamento físico não afetou a frequência de crises e concentração das DAEs.

Vale destacar que uma das limitações do estudo de Heise et al. (63) é que a maioria dos participantes estava livre de crises. Isso pode ser um fator limitante da aplicabilidade dos resultados, mas ao mesmo tempo tem seu valor, pois o treinamento físico não aumentou a frequência de crises dos sujeitos. Observações semelhantes quanto às características da amostra foram feitas por Nakken (45) e McAuley et al. (72). Pessoas com epilepsia refratária ao tratamento farmacológico naturalmente estão mais sujeitas a assumir um estilo de vida sedentário do que aqueles com epilepsias menos severas, assim como a terem crises associadas com a prática de atividade física. Portanto, estudos futuros deveriam ser feitos com a participação de pessoas com epilepsia ativa, definida como a ocorrência de no mínimo uma crise por mês.

\section{Epilepsia e a prática de atividade física esportiva}

Poucas pessoas com epilepsia praticam esporte em alto nível competitivo. Em uma pesquisa realizada com 6.000 estudantes colegiais, observou-se que apenas 17 tinham epilepsia e nenhum deles praticava esportes. Dos 3.000 atletas avaliados da National Collegiate Athletic Association (EUA), apenas um ginasta tinha epilepsia. Os resultados de um questionário enviado para treinadores da National Football League (NFL), National Basketball Association (NBA) e American Baseball League, na década de 70 do século XX, mostraram que apenas nove atletas com epilepsia eram atletas profissionais (73). Entretanto, alguns atletas famosos no passado assumiram publicamente que tinham epilepsia. Entre eles destacam-se: Hal Lanier, jogador de beisebol do San Francisco Giants e do New York Giants nas décadas de 60 e 70; Tony Lazzeri, jogador de beisebol dos Yankees; Grover Cleveland Alexander, jogador de beisebol do Philadelphia Phillies, Chicago Cubs e St. Louis Cardinals $(36,74)$; e mais recentemente Marion Clignet, ciclista francesa, medalhista nos jogos olímpicos de Atlanta, em 1996.

É importante considerar que a prática esportiva pode exacerbar as crises, porque se associa a fatores que alterariam o limiar para a ocorrência dessas crises, como o trauma repetitivo na cabeça durante a prática de esportes de contato, o exercício aeróbio feito em excesso, a hiperventilação decorrente do estresse emocional, o estresse físico e psicológico e alterações no metabolismo das DAEs (75). No entanto, os profissionais que prescrevem exercício físico devem saber que, respeitando-se algumas restrições, pessoas com crises epilépticas podem participar da maioria das atividades esportivas, pois as crises raramente acontecem durante a prática da atividade em si, ocorrendo com maior frequência no seu término ou durante a recuperação do estímulo $(9,39)$.

Ao longo do tempo, as recomendações para a participação esportiva de pessoas com epilepsia têm mudado (75). A American Medical Association Committee on the Medical Aspects of Sport $(76,77)$, bem 
como outras associações, recomendaram que uma pessoa com epilepsia de difícil controle farmacológico deveria evitar não somente esportes que envolvessem colisão e contato, como também aqueles que não envolvessem contato direto, como o tênis. Entretanto, em 1974 a mesma associação alterou seu posicionamento, afirmando que pessoas com epilepsia com moderado controle das crises poderiam praticar qualquer esporte, exceto atividades nas quais pudesse ocorrer trauma crônico da cabeça (78).

Em 1983, a American Academy of Pediatrics Committee on Children with Handicaps and Committee on Sports Medicine admitiu que se considerassem aspectos individuais, afirmando que a epilepsia por si não deveria excluir uma criança da prática do basquete, do hóquei, do futebol americano e da luta greco-romana. Entretanto, eles recomendaram que se evitasse a ginástica olímpica por causa do risco de queda. A Ilae recomendou que os esportes absolutamente proibidos para pessoas com epilepsia refratária ao tratamento farmacológico sejam o paraquedismo e o mergulho autônomo (79), pois essas pessoas têm quatro vezes mais chance de se envolver em acidentes com atividades que exigem submersão e que podem resultar em afogamento (80). Além do mais, esportes praticados nas alturas (alpinismo) ou em altas velocidades (automobilismo) conduzem tanto o participante quanto os espectadores a um risco aumentado de acidentes (7).

Portanto, alguns cuidados especiais devem ser levados em consideração para a prática esportiva por pessoas com epilepsia. A prática de algumas atividades de alto risco é contraindicada, como os esportes que envolvem risco de queda (alpinismo, barras paralelas, corrida de motocicleta e salto com paraquedas); outras requerem supervisão, pois envolvem risco moderado (natação, ciclismo, esqui aquático, canoagem, surfar, velejar e esportes que exigem cavalgar); para outras atividades o risco é baixo (prática de corrida de longa distância, como, por exemplo, a maratona); e finalmente há os que o risco é pouco conhecido, como os esportes de contato (futebol, futebol americano, basquete e beisebol) (36).

Além das crises, outro fator que poderia prejudicar o desempenho físico e o estado de consciência necessário para a prática de certos esportes, resultando em danos, seria o tratamento com as DAEs. Quando da prescrição de DAEs para esportistas, potenciais efeitos colaterais devem ser considerados. $\mathrm{O}$ fenobarbital e a primidona, por exemplo, têm efeitos sedativos, os quais podem ser prejudiciais para a participação esportiva. A fenitoína e a carbamazepina podem causar ataxia. Adicionalmente, um efeito colateral comum de algumas DAEs é o ganho de massa corporal, como é o caso do ácido valproico $(72,81)$, prejudicando o desempenho físico. A maioria das DAEs é permitida pelo Comitê Olímpico Internacional (COI), com algumas restrições nos esportes de tiro (25).

\section{Considerações finais}

Os efeitos da prática de atividade física regular por pessoas com epilepsia são incontestáveis. Essas pessoas podem ter os mesmos benefícios da sua prática que sujeitos saudáveis, tais como a melhora da aptidão cardiorrespiratória e muscular. Além disso, o exercício físico (agudo e/ou crônico) confere proteção contra o desencadeamento de crises, tendo em vista que trabalhos demonstraram de forma definitiva que as descargas epileptiformes no EEG diminuem, sendo poucos os casos documentados na literatura em que o desencadeamento da crise foi atribuído ao esforço físico. Evidentemente que a prática de algumas modalidades esportivas requer cuidados especiais, sobretudo, aqueles esportes com risco de trauma e afogamento.

\section{Agradecimentos}

Rodrigo Luiz Vancini foi bolsista de doutorado do Conselho Nacional de Desenvolvimento Científico e Tecnológico (CNPq).

\section{Referências}

1. Baker GA, Jacoby A, Buck D, Stalgis C, Monnet D. Quality of life of people with epilepsy: a European study. Epilepsia. 2005;46(1):132-40.

2. Brodie MJ, Schachter SC. Fast facts: epilepsy. 2a ed. Oxford: Health Press; 2001.

3. Borges MA, Min LL, Guerreiro CA, Yacubian EM, Cordeiro JA, Tognola WA, et al. Urban prevalence of epilepsy: populational study in Sao José do Rio Preto, a medium-sized city in Brazil. Arq Neuropsiquiatr. 2004;62(2A):199-204. 
4. Fisher RS, van Emde BW, Blume W, Eleger C, Genton P, Lee P, et al. Epileptic seizures and epilepsy: definitions proposed by the ILAE and the International Bureau for Epilepsy (IBE). Epilepsia. 2005;46(4):470-2.

5. Proposal for revised classification of epilepsies and epileptic syndromes. Commission on Classification and Terminology of the International League Against Epilepsy. Epilepsia. 1989;30(4):389-99.

6. Engel J Jr. Introduction to temporal lobe epilepsy. Epilepsy Res. 1996;26(1):141-50.

7. Howard GM, Radloff M, Sevier TL. Epilepsy and sports participation. Curr Sports Med Rep. 2004;3(1):15-9.

8. Engel J Jr. Concepts of epilepsy. Epilepsia. 1995;36: 23-9.

9. Bloomquist LEC. Epilepsy. In: ACSM's exercise management for persons with chronic diseases and disabilities. 2a ed. Champaign, IL: Human Kinetics; 2003. p. 262-6.

10. Temkin NR, Davis GR. Stress as risk factors for seizures among adults with epilepsy. Epilepsia 1984;25(4):450-6.

11. O'Donohoe NV. Epilepsies of childhood. 2a ed. London: Butterworth; 1985.

12. McLaurin R. Epilepsy and contact sports: factors contraindicating participation. JAMA. 1973;225(3):285-7.

13. Noakes TD, Goodwin N, Rayner BL, Branken T, Taylor RK. Water intoxication: a possible complication during endurance exercise. Med Sci Sports Exerc. 1985; 17(3):370-5.

14. Gates JR, Spiegel RH. Epilepsy, sports and exercise. Sports Med. 1993;15(1):1-5.

15. Speedy DB, Rogers I, Safih S, Foley B. Hyponatremia and seizures in an ultradistance triathlete. J Emerg Med. 2000;18(1):41-4.

16. Dimeff RJ. Seizure disorder in a professional American football player. Curr Sports Med Rep. 2006;5(4):173-6.

17. Millington JT. Should epileptics scuba dive? Correspondence. JAMA. 1985;254(22):3182-3.

18. French JK, Frengley PA. Hypoglycemia-induced seizures following a marathon. NZ Med J. 1983;96(732):407.

19. Esquivel E, Chaussain M, Plouin P, Ponsot G, Arthuis M. Physical exercise and voluntary hyperventilation in childhood absence epilepsy. Electroenceph Clin Neurophysiol 1991;79(2):127-32.
20. Osuntokun BO, Adeuja AOG, Nottidge VA, Schoenberg BS. Prevalence of the epilepsies in Nigerian Africans: a community-based study. Epilepsia. 1987;28:272-9.

21. Ablah E, Haug A, Konda K, Tinius AM, Ram S, Sadler T, et al. Exercise and epilepsy: a survey of Midwest epilepsy patients. Epilepsy Behav. 2009;14(1):162-6.

22. Robinson R. Cost benefit analisys. BMJ. 1993; 307 (6907):793-5.

23. Pato-Pato A, Cimas-Hernando I, Lorenzo-González JR, Vadillo-Olmo FJ. The economic impact of epilepsy. Rev Neurol. 2004;39(5):450-3.

24. Wiebe S. Brain surgery for epilepsy. Lancet. 2003; 362(Suppl):s48-9.

25. Cantu RV. Epilepsy and athletics. Clin Sports Med. 1998;17(1):61-9.

26. Duncan JS, Sander JW, Sisodiya SM, Walker MC. Adult epilepsy. Lancet. 2006;367(9516):1087-100.

27. Setkowicz Z, Mazur A. Physical training decreases susceptibility to subsequent pilocarpine-induced seizures in the rat. Epilepsy Res. 2006;71(2-3):142-8.

28. Arida RM, Cavalheiro EA, da Silva AC, Scorza FA. Physical activity and epilepsy: proven and predicted benefits. Sports Med. 2008(7);38:607-15.

29. Arida RM, Scorza FA, Scorza CA, Cavalheiro EA. Is physical activity beneficial for recovery in temporal lobe epilepsy? Evidences from animal studies. Neurosci Biobehav Rev 2009;33(3):422-31.

30. Arida RM, de Jesus Vieira A, Cavalheiro EA. Effect of physical exercise on kindling development. Epilepsy Res. 1998;30(2):127-32.

31. Arida RM, Scorza FA, dos Santos NF, Peres CA, Cavalheiro EA. Effect of physical exercise on seizure occurrence in a model of temporal lobe epilepsy in rats. Epilepsy Res. 1999;37(1):45-52.

32. Arida RM, Fernandes MJ, Scorza FA, Preti SC, Cavalheiro EA. Physical training does not influence interictal LCMRglu in pilocarpine-treated rats with epilepsy. Physiol Behav. 2003a;79(5-5):789-94.

33. Arida RM, Sanabria ER, da Silva AC, Faria LC, Scorza FA, Cavalheiro EA. Physical training reverts hippocampal electrophysiological changes in rats submitted to the pilocarpine model of epilepsy. Physiol Behav. 2004;83(1):165-71. 
34. Arida RM, Silva AV, Priel MR, Cavalheiro EA. Animals models of epilepsy. In: Turgut T, Fisher M. Handbook of experimental neurology. Cambridge, UK: Cambridge University Press; 2006. p. 438-56.

35. Arida RM, Scorza CA, Scorza FA, Gomes da Silva S, da Graça Naffah-Mazzacoratti M, Cavalheiro EA. Effects of different types of physical exercise on the staining of parvalbumin-positive neurons in the hippocampal formation of rats with epilepsy. Prog Neuropsychopharmacol Biol Psychiatry. 2007;31(4):814-22.

36. Dubow JS, Kelly JP. Epilepsy in sports and recreation. Sports Med. 2003;33(7):499-516.

37. Vieira D, Scorza FA, da Silva AC, Andrade MS, Cavalheiro EA, de Albuquerque $M$, et al. Efeitos benéficos do exercício físico nas epilepsias: o judô faz parte deste contexto? J Epilepsy Clin Neurophysiol. 2007;13:131-6.

38. Bennett DR. Epilepsy and the athlete. In: Jordan BD, Tsairis P, Warrent RF. Sports neurology. Rockville: Aspen; 1989. p. 116-26.

39. van Linschoten R, Backx FJ, Mulder OG, Meinardi H. Epilepsy and sports. Sports Med. 1990;10(1):9-19.

40. Denio LS, Drake Jr ME, Pakalnis A. The effect of exercise on seizure frequency. J Med. 1989;20(20):171-6.

41. Bjørholt PG, Nakken KO, Røhme K, Hansen H. Leisure time habits and physical fitness in adults with epilepsy. Epilepsia. 1990;31(1):83-7.

42. Roth DL, Goode KT, Williams VL, Faught E. Physical exercise, stressful life experience, and depression in adults with epilepsy. Epilepsia. 1994;35(6): 1248-55.

43. Steinhoff BJ, Neususs K, Thegeder H, Reimers CD. Leisure time activity and physical fitness in patients with epilepsy. Epilepsia. 1996;37(12):1221-7.

44. Jalava M, Sillanpaa M. Physical activity, healthrelated fitness, and health experience in adults with childhood-onset epilepsy: a controlled study. Epilepsia. 1997;38(4):424-9.

45. Nakken KO. Physical exercise in outpatients with epilepsy. Epilepsia. 1999;40(5):643-51.

46. Millett CJ, Johnson AL, Thompson PJ, Fish DR. A study of the relationship between participation in common leisure activities and seizure occurrence. Acta Neurol Scand. 2001;103(5):300-3.
47. Arida RM, Scorza FA, Albuquerque M, Cysneiros RM, de Oliveira RJ, Cavalheiro EA. Evaluation of physical exercise habits in Brazilian patients with epilepsy. Epilepsy Behav. 2003b;4(5):507-10.

48. Wong J, Wirrell E. Physical activity in children/teens with epilepsy compared with that in their siblings without epilepsy. Epilepsia. 2006;47(3):631-9.

49. Elliott JO, Lu B, Moore JL, McAuley JW, Long L. Exercise, diet, health behaviors, and risk factors among persons with epilepsy based on the California Health Interview Survey, 2005. Epilepsy Behav. 2008;13(2):307-15.

50. Vancini RL, Lira CAB, da Silva SG, Scorza FA, da Silva AC, Vieira D, et al. Evaluation of physical educators' knowledge about epilepsy. Arq Neuropsiquiatr. 2010; 68(3):367-71.

51. Nakken KO, Løyning A, Løyning T, Gløersen G, Larsson PG. Does physical exercise influence the occurrence of epileptiform EEG discharges in children? Epilepsia. 1997;38(3):279-84.

52. Eriksen HR, Ellertsen B, Grønningsaeter H, Nakken KO, Løyning Y, Ursin H. Physical exercise in women with intractable epilepsy. Epilepsia. 1994;35(6):1256-64.

53. Camilo F, Scorza FA, de Albuquerque M, Vancini RL, Cavalheiro EA, Arida RM. Evaluation of intense physical effort in subjects with temporal lobe epilepsy. Arq Neuropsiquiatr. 2009;67(4):1007-12.

54. Vancini RL, de Lira CA, Scorza FA, de Albuquerque M, Sousa BS, de Lima C, et al. Cardiorespiratory and electroencephalographic responses to exhaustive acute physical exercise in people with temporal lobe epilepsy. Epilepsy Behav. 2010;19(3):504-8.

55. Ogunyemi AO, Gomez MR, Klass DW. Seizures induced by exercise. Neurology. 1988;38(4):633-4.

56. Schmitt B, Thun-Hohenstein L, Vontobel H, Boltshauser E. Seizures induced by physical exercise: report of two cases. Neuropediatrics. 1994;25(1):51-3.

57. Sturm JW, Fedi M, Berkovic SF, Reutens DC. Exerciseinduced temporal lobe epilepsy. Neurology. 2002; 59(8):1246-8.

58. Werz MA. Idiopathic generalized tonic-clonic seizures limited to exercise in a young adult. Epilepsy \& Behavior. 2005;6(1):98-101. 
59. Götze W, Kubicki S, Munter M, Teichmann J. Effect of physical exercise on seizure threshold (investigated by electroencephalographic telemetry). Dis Nerv Syst. 1967;28(10):664-7.

60. Sahoo SK, Fountain NB. Epilepsy in football players and other land-based contact or collision sport athletes: when can they participate, and is there an increased risk? Curr Sports Med Rep. 2004;3(5):284-8.

61. Frucht MM, Quigg M, Schwaner C, Fountain NB. Distribution of seizure precipitants among epilepsy syndromes. Epilepsia. 2000;41(12):1534-9.

62. Simpson Jr RK, Grossman RG. Seizures after jogging. N Engl J Med. 1989;321(12):835.

63. Heise J, Buckworth J, McAuley JW, Long L, Kirby TE. Exercise training results in positive outcomes in persons with epilepsy. Clin Exerc Physiol. 2002;4:79-84.

64. Morgan WP. Affective beneficence of vigorous physical activity. Med Sci Sports Exerc. 1985;17(1):94-100.

65. Albrecht H. Endorphins, sport, and epilepsy: getting fit or having one. NZ Med J. 1986;99(814):915.

66. Gehlert S. Perceptions of control in adults with epilepsy. Epilepsia. 1994;35(1):81-8.

67. American Academy of Pediatrics Committee on Children with Handicaps and Committee on Sports Medicine. Sports and the child with epilepsy. Pediatrics. 1983;72(6):884-5.

68. Kuijer A. Epilepsy and exercise, electroencephalographical and biochemical studies. In: Wada JA, Penry JK. Advances in Epileptology: Xth Epilepsy International Symposium. New York: Raven Press, 1980. p. 543.

69. Nakken KO, Bjørholt PG, Johannessen SI, Løyning T, Lind E. Effect of physical training on aerobic capacity, seizure occurrence, and serum level of antiepiLevine S, Ursin H. What is stress? In: Brown MR, Koob GF, Rivier C. Stress-neurobiology and neuroendocrinology. New York: Marcel Dekker; 1991. p. 3-21.

70. van Doornen LJ, de Geus EJ, Orlebeke JF. Aerobic fitness and the physiological stress response: a critical evaluation. Soc Sci Med. 1988;26(3):303-7.
71. McAuley JW, Long L, Heise J, Kirby T, Buckworth J, Pitt C, et al. A prospective evaluation of the effects of a 12-week outpatient exercise program on clinical and behavioral outcomes in patients with epilepsy. Epilepsy Behav. 2001;2(6):592-600.

72. Bennett DR. Sports and epilepsy: to play or not to play. Semin Neurol. 1981;1:345-57.

73. Chase D. With epilepsy, they take the medicine and play. Phys Sportsmed. 1974;2:58-61.

74. Fountain NB, May AC. Epilepsy and athletics. Clin Sports Med. 2003;22(3):605-16.

75. American Medical Association Committee on the Medical Aspects of Sports. Convulsive disorders and participation in sports and physical education. JAMA. 1968;206(6):1291.

76. American Medical Association Committee on the Medical Aspects of Sports The epileptic child and competitive school athletics. Pediatrics. 1968;42:700-2.

77. Corbitt RW, Cooper DL, Erickson DJ, Kriss FC, Thornton ML, Craig TT. Editorial: Epileptics and contact sports. JAMA. 1974;229(7):820-1.

78. ILAE Commission report. Restrictions for children with epilepsy. Commission of Pediatrics of the ILAE International League Against Epilepsy. Epilepsia. 1997; 38(9):1054-6.

79. O'Donohoe NV. What should the child with epilepsy be allowed to do? Arch Dis Child. 1983;58(11):934-7.

80. Corman CL, Leung NM, Guberman AH. Weight gain in epileptic patients during treatment with valproic acid: a retrospective study. Can J Neurol. 1997;24(3): 240-4.
Recebido: $11 / 01 / 2011$

Received: 01/11/2011

Aprovado: 18/03/2011

Approved: 03/18/2011 